\title{
Risk-Averse Outsourcing Strategies for Optimal Intellectual Property Protection
}

\author{
Baejin Kim, Karim Hamza, and Kazuhiro Saitou, Senior Member, IEEE
}

\begin{abstract}
This paper presents an extension of our previous work on optimal allocation of external suppliers to manage the risk of leakage in the intellectual property (IP) of a product. The optimal outsourcing decision problem is modeled as a bi-objective minimization problem with one objective being the estimated risk of loss of IP of a product due to outsourcing (IP leakage) and another being manufacturing cost of the product. A number of extensions are made in the problem formulation, including 1) unified probabilistic modeling of IP loss, 2) consideration of manufacturing process sequence to model the effect of partial observation on the compromise by a supplier, 3 ) modeling nonlinear effects on the increase in the probability of compromise by a supplier performing (and observing) multiple manufacturing processes, and 4) generalization to multi-supplier cases. A case study on a hinge mechanism for a cellular phone is presented, where a multi-objective genetic algorithm is used to generate the Pareto optimal supplier allocations exhibiting the trade-offs between the two objectives.
\end{abstract}

\section{INTRODUCTION}

$\mathrm{O}$ UTSOURCING is a practice often used by companies to reduce the in-house manufacturing costs, thereby maximizing profitability and maintaining competitive edge in the market. These benefits can be greatly undermined if the outsourced suppliers leak the intellectual property (IP) of the products to the competitors, who can produce unlawful imitation of the products. According to the Office of the United States Trade Representative, IP-theft worldwide costs American companies $\$ 250$ billion a year [1].

Prevention of IP leakage through the outsourced suppliers is not an easy task. While original equipment manufacturers (OEMs) typically put in place outsourcing contracts that seek to inhibit IP leakage, the OEM rarely have direct control on the IP security mechanisms at the suppliers' end. It is therefore of great value for OEMs to perform risk assessment of IP leakage in spite of the leakage inhibiting mechanisms being in place. Such assessment would allow OEMs to selectively manage the risk of IP-leak while enjoying the benefit of cost saving through outsourcing [2].

Suppliers can compromise the information associated with the outsourced parts and manufacturing processes by imitating the parts and/or the processes without permission of OEM or by providing the information to the competitors of OEM. In either case, simply compromising the information is not necessarily damage the IP value of the product. If the parts and processes do not play important roles in the IP value

Manuscript received June 15, 2010. B. Kim, K. Hamza, and K. Saitou are with the Department of Mechanical Engineering, University of Michigan, Ann Arbor, MI 48109, USA (e-mail:, \{baekim, khamza, kazu\}@umich.edu). of the product, the damage of such compromise is minimal and perhaps the economical advantage of outsourcing would overrule the potential risk of IP leak. On the other hand, if the parts and processes play key roles in the IP value of the product, the potential damage of compromise can be so large that it overrules economical advantage of outsourcing. Therefore, optimal decision on outsourcing depends on 1) how likely a supplier compromises the outsourced parts and manufacturing processes, and 2) how likely the compromise leads to the damage in the IP value of the product. We assume 1) can be estimated by historical data of a supplier and 2) can be estimated by textual analysis of patent claims of the products and insights from experts.

This paper presents an extension of our previous work [3], where the optimal outsourcing decision problem is modeled as a bi-objective optimization problem with one objective being the estimated risk of loss of IP of a product due to outsourcing and the other being manufacturing cost of the product. While loss of IP causes financial damage and thus ultimately is a form of cost, quantification of the cost of IP leakage is a very complicated task that is subject to many unpredictable and/or unforeseeable factors, hence our treats risk of IP leakage as a separate objective than the manufacturing cost. A number of extensions are made in the problem formulation, including 1) unified probabilistic modeling of IP loss, 2) consideration of manufacturing process sequence to model the effect of partial observation on the compromise by a supplier, and 3) modeling nonlinear effects on the increase in the probability of compromise by a supplier performing (and observing) multiple manufacturing processes, and 4) generalization to multi-supplier cases. A case study on a hinge mechanism for a cellular phone is presented, where a multi-objective genetic algorithm [4] is used to generate the Pareto optimal supplier allocations exhibiting the trade-offs between the two objectives.

\section{RELATED WORK}

Due to its relevance to modern economy driven by global supply chain networks, supplier selection problems (also known as vendor selection problems) have been extensively studied by the operations research community. A classic work by Dickson [5] identified 23 criteria for supplier selection, with top 5 being price, quality, lead-time, technical service, and delivery reliability. Later studies found these criteria are still applicable for global supply chains [6,7]. These criteria serve as basis of multi-criteria supplier evaluation/selection methods such as data envelopment analysis (DEA) [8-10], analytical hierarchical processes (AHP) [11-13], and 
multi-objective optimization [14-17]. Researchers also have expanded the scope of supplier selection problems to integrate the models of volume discount [18-20], inventory management [21, 22], supplier sync and just-in-time delivery $[23,24]$, and multiple products and product life cycles [25-27]. These work, however, do not incorporate the risk of IP leakage from suppliers as a decision criterion.

While many papers reported management strategies for information protection in global supply chain network [28-31], they are on guidelines and best practices, and do not provide quantitative supplier selection criteria to prevent IP leakage nor mathematical modeling of the risk of IP leak. To the best of our knowledge, there has not been any work on mathematical approaches for supplier selection to prevent IP leakage other than our previous work [3]. Previous work in the literature views outsourcing as a binary decision: outsource a part to a supplier or not. Our method, on the other hand, considers partial outsourcing as possible options, where multiple non-IP critical manufacturing processes on the same part can be outsourced to different suppliers while IP-critical processes are kept in-house.

\section{METHOD}

Consider an example garden-care product consisting attachment head and ground piece, with each part having an additional feature as in Fig. 1a. The manufacturing process tree for the product (Fig. 1.b) consists of five processes:

- Process $p_{1}$ : manufacturing of base part of the head piece

- Process $p_{2}$ : manufacturing of hole feature on head piece

- Process $p_{3}$ : manufacturing of base part of ground piece

- Process $p_{4}$ : manufacturing of pin feature on ground piece

- Process $p_{5}$ : assembly of completed parts

Let us assume the product also has three patentable claims as listed in Table I. An example outsourcing scenario of this product is where Process $p_{1}$ is outsourced, but Process $p_{2}$ is done in-house at a later stage, along with Processes $p_{3}, p_{4}$ and $p_{5}$. This scenario takes advantage of low manufacturing cost for process $p_{1}$, while reducing the risk of IP leakage associated with claim 3. This is because features associated with claim 3 (hole and pin) are manufactured in-house. We will use this example to illustrate our optimization model.

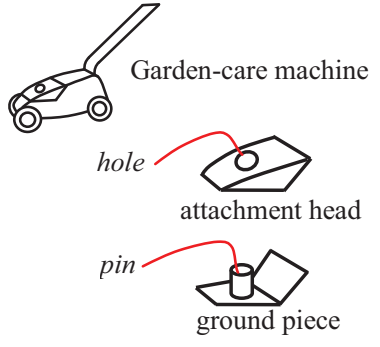

(a)

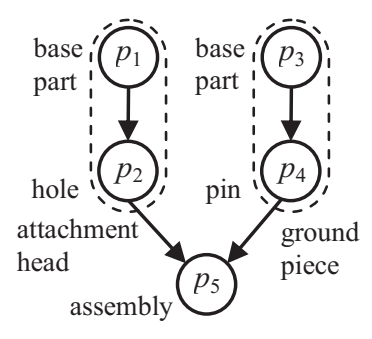

(b)
Fig. 1 example garden-care product: (a) parts and features, (b) manufacturing process tree.

\section{A. Assumptions}

We consider a product composed of a number of parts. A part is composed of a base part and features (as in Fig 1a), each of which can be manufactured in-house or by different suppliers. The following is assumed:
- Manufacturing process plan of the product is given as a process tree consisting of $n_{p}$ processes $p_{i}, i=1,2, \ldots, n_{p}$.

- Intellectual properties associated with the product are documented as claim $c_{k}, k=1,2, \ldots, n_{c}$, in a patent document (draft or approved).

- $\quad$ Outsourcing base parts or features to supplier $s_{l}, l=2$, $3, \ldots, n_{s}$, reduces their manufacturing costs at some rate compared to in-house manufacturing (denoted as $s_{1}$ ).

- A process can be outsourced to only one supplier, whereas one supplier can perform multiple processes.

- IP leakages occur only through the compromises of manufacturing processes by suppliers.

- The compromise of a manufacturing process is caused only by 1) a supplier who performs the process, or 2) a supplier who receives the parts after the process is performed (i.e., a supplier who performs a downstream process in the process tree). This means a process can possibly be compromised by multiple suppliers.

- The IP compromise of a process is independent from the IP compromise of another manufacturing process, even if they are caused by the same supplier.

- One incidence of the IP leakage associated with a claim by a supplier completely destroys the IP of the claim; another IP leakage incidence by another supplier does not do any additional harm.

TABLE I

PATENT CLAIMS OF GARDEN-CARE PRODUCT

\begin{tabular}{cl}
\hline \hline Claim & \multicolumn{1}{c}{ Description } \\
\hline 1 & $\begin{array}{l}\text { An attachment head attaches to a lawn-mowing machine } \\
\text { A ground piece performs a weed-disinfection operation during } \\
\text { mowing }\end{array}$ \\
3 & $\begin{array}{l}\text { A pin-feature on the ground piece has a mechanism that engage } \\
\text { with a hole-feature on the attachment head that allows for safe } \\
\text { operation and detachment }\end{array}$ \\
\hline \hline
\end{tabular}

\section{B. Model Parameters and Design Variables}

We refer $P$ as a set of manufacturing processes in the process tree, $C$ as a set of claims in the patent document, and $S$ is a set of available suppliers. The design variable $\boldsymbol{x}=\left(x_{1}, x_{2}\right.$, $\left.\ldots, x_{n p}\right)$ is the outsourcing decisions associated with each process, where $x_{i} \in\left\{1, \ldots, n_{s}\right\}$ is the index of the supplier chosen to perform the manufacturing process $p_{i}$. This representation ensures each process is outsourced to only one supplier. The optimal outsourcing decision problem is posed as a bi-objective optimization problem as:

$$
\begin{aligned}
& \underset{x}{\operatorname{minimize}} f_{1}(\boldsymbol{x}), f_{2}(\boldsymbol{x}) \\
& \text { subject to } \boldsymbol{x} \in\{1,2, \ldots
\end{aligned}
$$

where $f_{1}(\boldsymbol{x})$ is an objective function estimating the loss of intellectual property of the product and $f_{2}(\boldsymbol{x})$ is an objective function estimating the manufacturing cost of the product, according to the outsourcing decisions specified by $\boldsymbol{x}$.

Based on the assumption that one incidence of the IP leakage associated with a claim completely destroys the IP of the claim, we define $f_{1}(\boldsymbol{x})$ as weighted sum of the maximum probabilities such incidence over all claims:

$$
f_{1}(\boldsymbol{x})=\sum_{k=1}^{n_{C}} w_{k} \times \phi_{k}
$$


where $w_{k} \in[0,1]$ is the (estimated) fraction of the IP value of claim $c_{k}$ in the IP of an entire product (i.e., all claims) and $\phi_{k}$ is the probability that IP of claim $c_{k}$ leaks from the suppliers that have manufactured or seen parts of the product:

$$
\phi_{k}(\boldsymbol{x})=\operatorname{Pr}(\bigsqcup
$$

where $A_{k l}$ be an event that IP of claim $c_{k}$ leaks from supplier $s_{l}$. Since events $A_{k l}, 1=1, \ldots, n_{S}$ are generally not disjoint (i.e., multiple suppliers can leak the IP of the same claim), $\phi_{k}$ can be computed recursively as:

$$
\begin{gathered}
\phi_{k}(\boldsymbol{x})=\operatorname{Pr}\left(E_{k n_{S}}\right) \\
\operatorname{Pr}\left(E_{k l}\right)=\left\{\begin{array}{cc}
\operatorname{Pr}\left(E_{k l-1}\right)+\operatorname{Pr}\left(A_{k l}\right)-\operatorname{Pr}\left(E_{k l-1} \cap A_{k l}\right) & l \geq 2 \\
\operatorname{Pr}\left(A_{k l}\right) & l=1
\end{array}\right.
\end{gathered}
$$

where $E_{k l}=A_{k 1} \cup A_{k 2} \cup \ldots A_{k l}$. Since that IP of claim $c_{k}$ leaks from supplier $s_{l}$ due to the compromise of any processes in the process tree, $\operatorname{Pr}\left(A_{k l}\right)$ in equation 5 is given as:

$$
\operatorname{Pr}\left(A_{k l}\right)=\operatorname{Pr}(\bigsqcup
$$

where $B_{k i l}$ is an event that IP of claim $c_{k}$ leaks due to the compromise of process $p_{i}$ by supplier $s_{l}$. Since events $B_{k i l}, i=$ $1, \ldots, n_{P}$ are generally not disjoint (i.e., compromises of multiple processes can cause the leak of the same claim), $\operatorname{Pr}\left(A_{k l}\right)$ can also be computed recursively in a similar manner to $\phi_{k}$, using an individual event probability:

$$
\operatorname{Pr}\left(B_{k i l}\right)=\omega_{k i} \times \theta_{i l}(\boldsymbol{x})
$$

where $\omega_{k i} \in[0,1]$ is the probability that IP of claim $c_{k}$ leaks given the compromise of process $p_{i}$, and $\theta_{i l}(\boldsymbol{x}) \in[0,1]$ is the probability that process $p_{i}$ is compromised by supplier $s_{l}$.

We assume probability $\omega_{k i}$ can be estimated by analyzing the association between claim $c_{k}$ and process $p_{i}$ in the patent document and insights from experts. The association between a process and a claim is determined as follows: Process $p_{i}$ is associated with claim $c_{k}$ if 1) $p_{i}$ manufactures any feature mentioned in $c_{k}$,2) $p_{i}$ assembles any part mentioned in $c_{k}$, or 3 ) $p_{i}$ assembles part(s) with any feature mentioned in $c_{k}$. Naturally, $\omega_{k i}=0$ if process $p_{i}$ is not associated with claim $c_{k}$, and $\omega_{k i} \in(0,1]$ if process $p_{i}$ is associated with claim $c_{k}$, in which case the numerical value must be estimated by experts based on her engineering knowledge.

Since a supplier can compromise a process by performing it or by "seeing" it (a supplier performs a downstream process in the process tree), probability $\theta_{i l}(\boldsymbol{x})$ is given as:

$$
\theta_{i l}(\boldsymbol{x})=\alpha_{i l}(\boldsymbol{x}) \times \delta_{i l}(\boldsymbol{x})+\beta_{i l}(\boldsymbol{x}) \times \gamma_{i l}(\boldsymbol{x})
$$

where $\alpha_{i l}(\boldsymbol{x}) \in[0,1]$ is the probability that process $p_{i}$ is compromised if it is outsourced to supplier $s_{l}, \beta_{i l}(\boldsymbol{x}) \in[0,1]$ is the probability that process $p_{i}$ is compromised if it is "seen by" supplier $s_{l}$, and:

$$
\begin{gathered}
\delta_{i l}(\boldsymbol{x})=\left\{\begin{array}{lc}
1 & \text { if } l=x_{i} \\
0 & \text { otherwise }
\end{array}\right. \\
\gamma_{i l}(\boldsymbol{x})=\left\{\begin{array}{cc}
1 & \text { if } i \in Q_{l}(\boldsymbol{x}) \\
0 & \text { otherwise }
\end{array}\right.
\end{gathered}
$$

where $Q_{l}(\boldsymbol{x})$ is a set of the indices of the processes that are seen but not manufactured at supplier $s_{l}$, given as:

$$
Q_{l}(\boldsymbol{x})=\left(\bigcup_{i \in M_{l}(\boldsymbol{x})}, \quad \boldsymbol{x}\right)
$$

where $M_{l}(\boldsymbol{x})$ is a set of the indices of the processes that are outsourced to supplier $s_{l}$ and $U_{i}$ is a set of the indices of the manufacturing processes that are upstream of $p_{i}$ in the process tree of the product. The set $M_{l}(\boldsymbol{x})$ is given as:

$$
M_{l}(\boldsymbol{x})=\left\{i \mid p_{i} \in P, x_{i}=l\right\}
$$

Given the process tree as a directed graph, set $U_{i}$ can be computed as a set of nodes reachable from $p_{i}$ in the reverse direction of edges. Note that if $\delta_{i l}(\boldsymbol{x})=1$, then $\gamma_{i l}(\boldsymbol{x})=0$ and if $\gamma_{i l}(\boldsymbol{x})=1$, then $\delta_{i l}(\boldsymbol{x})=0$, since a process cannot be performed and seen by the same supplier.

A supplier is more likely to compromise processes if it is performing or seeing multiple processes associated with the same claim. We assume the increases of the probabilities $\alpha_{i l}(\boldsymbol{x})$ and $\beta_{i l}(\boldsymbol{x})$ are proportional to a power of the number of processes associated with the same claim as $p_{i}$ and performed or seen by $s_{l}$ :

$$
\begin{aligned}
& \alpha_{i l}(\boldsymbol{x})=\left(\alpha_{i l}^{\max }-\alpha_{i l}^{\min }\right) \operatorname{bow}\left(w_{1} v_{i l}(x)+w_{2} u_{i l}(x), \mu_{i l}\right)+\alpha_{i l}^{\min } \\
& \beta_{i l}(\boldsymbol{x})=\left(\beta_{i l}^{\max }-\beta_{i l}^{\min }\right) \operatorname{bow}\left(w_{1} v_{i l}(x)+w_{2} u_{i l}(x), \sigma_{i l}\right)+\alpha_{i l}^{\min }
\end{aligned}
$$

where $\operatorname{bow}()$ is a "bow"-shaped function, given by:

$$
\operatorname{bow}(y, r)=1-\left(1-y^{r}\right)^{1 / r}
$$

and $v_{i l}(\boldsymbol{x}) \in[0,1]$ is the fractions of the number of processes associated with the same claim as $p_{i}$ and performed by $s_{l}$, $u_{i l}(\boldsymbol{x}) \in[0,1]$ is the fractions of the number of processes associated with the same claim as $p_{i}$ and seen by $s_{l}, \alpha_{i l}{ }^{\mathrm{min}}$, $\alpha_{i l}{ }^{\max } \in[0,1]$ are the minimum and maximum values $\alpha_{i l}(\boldsymbol{x})$ can take, respectively, $\beta_{i l}{ }^{\min }, \beta_{i l}^{\max } \in[0,1]$ are the minimum and maximum values $\beta_{i l}(\boldsymbol{x})$ can take, respectively. $w_{\mathrm{v}}$ and $w_{\mathrm{u}}$ are weights for $v_{i l}(\boldsymbol{x})$ and $u_{i l}(\boldsymbol{x})$, respectively, and $\mu_{i l}, \sigma_{i l}>0$ are the powers of increase in $\alpha_{i l}(\boldsymbol{x})$ and $\beta_{i l}(\boldsymbol{x})$, respectively. Since performing a process should increase the probabilities of compromise more than seeing it, we should have $w_{\mathrm{v}} \leq w_{\mathrm{u}}$

The fractions $v_{i l}(\boldsymbol{x})$ and $u_{i l}(\boldsymbol{x})$ are given as

$$
\begin{aligned}
& v_{i l}(\boldsymbol{x})=\frac{1}{\left|B_{i}\right|} \sum_{k \in B_{i}} \frac{\left|R_{k} \cap M_{l}(\boldsymbol{x})\right|}{\left|R_{k}\right|} \\
& u_{i l}(\boldsymbol{x})=\frac{1}{\left|B_{i}\right|} \sum_{k \in B_{i}} \frac{\left|R_{k} \cap Q_{l}(\boldsymbol{x})\right|}{\left|R_{k}\right|}
\end{aligned}
$$

where $B_{i}$ is a set of the indices of the claims that are associated with process $p_{i}, R_{k}$ is a set of the indices of the processes associated with claim $c_{k}$. Sets $B_{i}, R_{k}$, are given as:

$$
\begin{aligned}
& B_{i}=\left\{k \mid c_{k} \in C, \omega_{k i} \neq 0\right\} \\
& R_{k}=\left\{i \mid p_{i} \in P, \omega_{k i} \neq 0\right\}
\end{aligned}
$$

The manufacturing cost objective $f_{2}(\boldsymbol{x})$ is defined as:

$$
f_{2}(\boldsymbol{x})=\sum_{i=1}^{n_{p}} a_{i} \times d_{i}(\boldsymbol{x}) \times e_{i}(\boldsymbol{x})
$$

where $a_{i} \in[0,1]$ is the fraction the manufacturing cost of process $p_{i}$ in the total manufacturing cost of the product, $d_{i}(\boldsymbol{x})$ $\in[0,1]$ is a discount factor of the supplier that performs process $p_{i}$, and $e_{i}(\boldsymbol{x}) \in[1, \infty)$ is a cost penalty factor for outsourcing related processes to different suppliers. Factor $e_{i}(\boldsymbol{x})$ is intended to model the fact that some features in one part are more economically manufactured together by one 
supplier via net forming processes, than manufactured separately by different suppliers:

$$
e_{i}(\boldsymbol{x})=\prod_{\substack{j \in\left\{1,2, \ldots n_{p}\right\} \\ x_{j} \neq x_{i}}} g_{i j}
$$

where $g_{i j}$ is a cost penalty on process $p_{i}$ if it is outsourced to a different supplier. It is typically possible to negotiate a better discount from a supplier by assigning them processes with higher values. We assume a linear model for the discount:

$$
d_{i}(\boldsymbol{x})=1-\left\{b_{x_{l}}(\boldsymbol{x}) \times\left(d_{x_{i}}^{\max }-d_{x_{i}}^{\min }\right)+d_{x_{i}}^{\min }\right\}
$$

where $d_{l}^{\min }$ and $d_{l}^{\max }$ are the minimum and maximum discounts obtainable from supplier $s_{l}$, respectively, and $b_{l}(\boldsymbol{x})$ is the fraction of total manufacturing cost assigned to $s_{l}$, as:

$$
b_{l}(\boldsymbol{x})=\sum_{p_{i} \in M_{l}(\boldsymbol{x})} a_{i}
$$

\section{Sample Results}

Consider the garden care product in Fig. 1 having the data:

- Two external suppliers are available $\left(n_{s}=3\right)$.

- In-house manufacturing $s_{1}$ is completely secure $\left(\alpha_{i 1}{ }^{\min }=\right.$ $\alpha_{i 1}{ }^{\max }=\mu_{i 1}=\sigma_{i 1}=\beta_{i 1}{ }^{\min }=\beta_{i 1}{ }^{\max }=0$ for all $i$ ).

- Supplier $s_{2}$ has $50-70 \%$ risk for processes manufactured $\left(\alpha_{i 2}{ }^{\min }=0.5\right.$ and $\left.\alpha_{i 2}{ }^{\max }=0.7\right)$ with $\mu_{i 2}=0.5$ and $\sigma_{i 2}=1$ for all $i$, and $25-50 \%$ risk for processes seen $\left(\beta_{i 2}{ }^{\min }=0.25\right.$ and $\left.\beta_{i 2}{ }^{\max }=0.5\right)$ with $\eta_{i 2}=0.5$ and $\rho_{i 2}=1$ for all $i$.

- Supplier $s_{3}$ has $40-50 \%$ risk for processes manufactured $\left(\alpha_{i 3}{ }^{\min }=0.4\right.$ and $\left.\alpha_{i 3}{ }^{\max }=0.5\right)$ with $\mu_{i 3}=0.5$ and $\sigma_{i 3}=1$ for all $i$, and $20-30 \%$ risk for processes seen $\left(\beta_{i 3}{ }^{\min }=0.2\right.$ and $\beta_{i 3}{ }^{\max }=0.3$ ) with $\eta_{i 3}=0.5$ and $\rho_{i 3}=1$ for all $i$.

- Weights in the bow function are $w_{v}=0.667$ and $w_{u}=0.333$

- The probabilities of IP leak of a claim given the compromise of a process $\left(\omega_{k i}\right)$ is shown in Table II.

- All claims are equally important $\left(w_{1}=w_{2}=w_{3}=0.333\right)$

- The cost fraction for a manufacturing process $\left(a_{i}\right)$ and the cost penalty for process separation $\left(g_{i j}\right)$ are shown in Fig. 3. All $g_{i j}$ 's are equal to 1 if not shown in Fig. 2.

- In-house manufacturing gets no discount $\left(d_{1}{ }^{\min }=d_{1}{ }^{\max }=\right.$ $0)$. Supplier $s_{2}$ gives $20-24 \%$ discount $\left(d_{2}{ }^{\text {min }}=0.2\right.$ and $\left.d_{2}{ }^{\max }=0.24\right)$. Supplier $s_{3}$ gives $16-20 \%$ discount $\left(d_{3}{ }^{\min }=\right.$ 0.16 and $d_{3}{ }^{\max }=0.2$ ).

TABLE II

PROBABILITY OF IP LEAK FOR GARDEN-CARE PRODUCT

\begin{tabular}{cccccc}
\hline \hline \multirow{2}{*}{ Claims } & \multicolumn{5}{c}{ Process } \\
& $P_{1}$ & $P_{2}$ & $P_{3}$ & $P_{4}$ & $P_{5}$ \\
\hline$c_{1}$ & 0.7 & 0 & 0 & 0 & 1.0 \\
$c_{2}$ & 0 & 0 & 0.7 & 0 & 1.0 \\
$c_{3}$ & 0 & 0.7 & 0 & 0.7 & 1.0 \\
\hline \hline
\end{tabular}

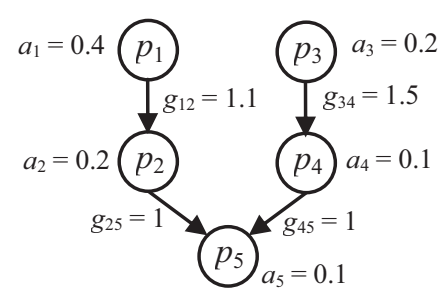

Fig. 2 Process cost fractions and separation penalty for garden care product

Since there are only 5 design variables in this example with 3 possible choices for each, the total number of possible outsourcing scenarios is $3^{5}=243$. This allows for generation and examination of all of them via exhaustive enumeration in order to generate the Pareto-plot in Fig. 3. The Pareto-plot shows the best possible outsourcing scenarios and their corresponding trade-off between the manufacturing cost and the risk of IP leakage. It should be noted that when considering products of realistic level of detail, the total number of all possible scenarios is $\left(s^{n}\right)$, which quickly becomes unreasonably large for full enumeration. However, only the Pareto-optimal scenarios are of interest. The case study presented in next section will use a multi-objective genetic algorithm (MOGA) [8] for exploration of the Pareto-optimal scenarios without need for full enumeration.

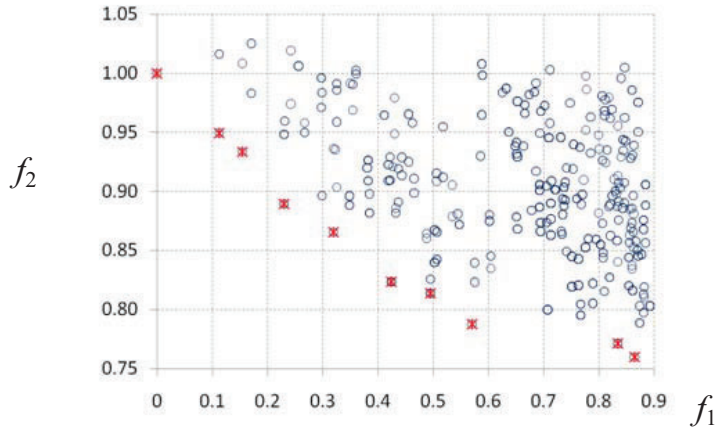

Fig. 3 Pareto-Optimal outsourcing scenarios for garden care product

\section{CASE Study}

\section{A. Problem Data}

A cellular phone slide hinge mechanism manufactured by LG Electronics (Fig. 4) $[32,33]$ is considered. The patent includes six main claims $\left(n_{\mathrm{c}}=6\right)$ as summarized in Table III.

Eleven manufacturing and assembly processes $\left(n_{p}=11\right)$ are considered in this study. The process sequence tree, fractional of total cost, and the feature separation penalty factors are shown in Fig. 5. Three external suppliers and in-house manufacturing are assumed (thus $n_{s}=4$ ). The patent claims are used to construct the process compromise risks in Table IV and the claim weights in Table V. In-house manufacturing is assumed to be completely safe but gets no discount. Values of the risk and discount parameters are listed in Table VI.

\section{B. Result and Discussion}

A Multi-objective genetic algorithm based on NSGA-II [34] is employed to generate the Pareto-optimal outsourcing scenarios shown in Fig. 6. Some note-worthy outsourcing scenarios from Fig. 6 are listed in Table VII. Qualitative analysis of these scenarios is summarized as:

Scenario a: All processes are manufactured in-house, which provides minimal risk of IP leakage, but maximal cost

Scenario b: All processes are outsourced to the supplier that provides the best discount. Note that both supplier 1 and 3 provide the same discount in this case study, but supplier 1 is perceived to be slightly more secure than supplier 3

Scenario c: Only a small number of the processes are outsourced. Additionally feature separation was employed in the elastic member (processes 7 and 8 outsourced to different suppliers). This results in a small reduction in cost at a small trade-off in risk of IP leakage 
Scenario d: Several processes are outsourced. However, the more critical ones are kept in-house. Supplier 2 which is perceived to be more secure gets more processes. It is also observed in this scenario that distributing processes to multiple suppliers reduces the overall risk of IP leakage even if some suppliers have higher risk (supplier 3 offers same discount as supplier 1, but has slightly higher risk) Scenario $e:$ All the processes are outsourced except the sub-assembly and final assembly.

Scenario f: All the processes are outsourced except final assembly.

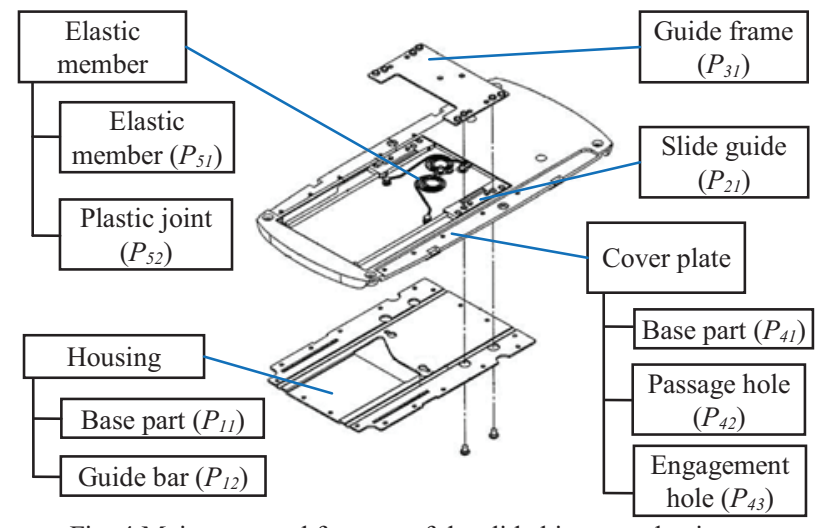

Fig. 4 Main parts and features of the slide hinge mechanism

TABLE III

Patent Claims of the Slide Hinge Mechanism

\begin{tabular}{|c|c|}
\hline Claim & Description \\
\hline 1 & $\begin{array}{l}\text { A slide part includes the housing }\left(P_{11}\right) \text { in which a setting hole is } \\
\text { formed in a back surface of the housing, and a slide guide }\left(P_{21}\right) \\
\text { sliding along the guide bar }\left(P_{12}\right) \text { mounted on the body part }\end{array}$ \\
\hline 2 & $\begin{array}{l}\text { A cover plate }\left(P_{41}\right) \text { combines with the inside of the housing } \\
\left(P_{I I}\right) \text { to cover the setting hole }\end{array}$ \\
\hline 3 & $\begin{array}{l}\text { A guide locking protrusion is formed on an outside surface of } \\
\text { the slide guide }\left(P_{2 l}\right) \text {, and a guide locking projection is formed } \\
\text { on an inside surface in the housing }\left(P_{11}\right) . P_{11} \text { and } P_{2 l} \text { lock }\end{array}$ \\
\hline 4 & $\begin{array}{l}\text { An elastic member }\left(P_{5 l}\right) \text { in which one end is connected to the } \\
\text { cover plate }\left(P_{4 l}\right) \text {, and the other end to the guide frame }\left(P_{3 l}\right) \text { so } \\
\text { that sliding of the slide part may be elastically performed }\end{array}$ \\
\hline 5 & $\begin{array}{l}\text { A passage hole }\left(P_{42}\right) \text { is formed in the cover plate so that a } \\
\text { flexible printed circuit board electrically connecting the body } \\
\text { part with the slide part may pass }\end{array}$ \\
\hline 6 & $\begin{array}{l}\text { An elastic member }\left(P_{51}\right) \text { connected with the cover plate }\left(P_{41}\right) \\
\text { includes a plastic jointer }\left(P_{52}\right) \text {, and an engagement hole }\left(P_{43}\right) \text { is } \\
\text { formed in the cover plate so that the plastic jointer may be } \\
\text { combined to perform a rotation }\end{array}$ \\
\hline
\end{tabular}

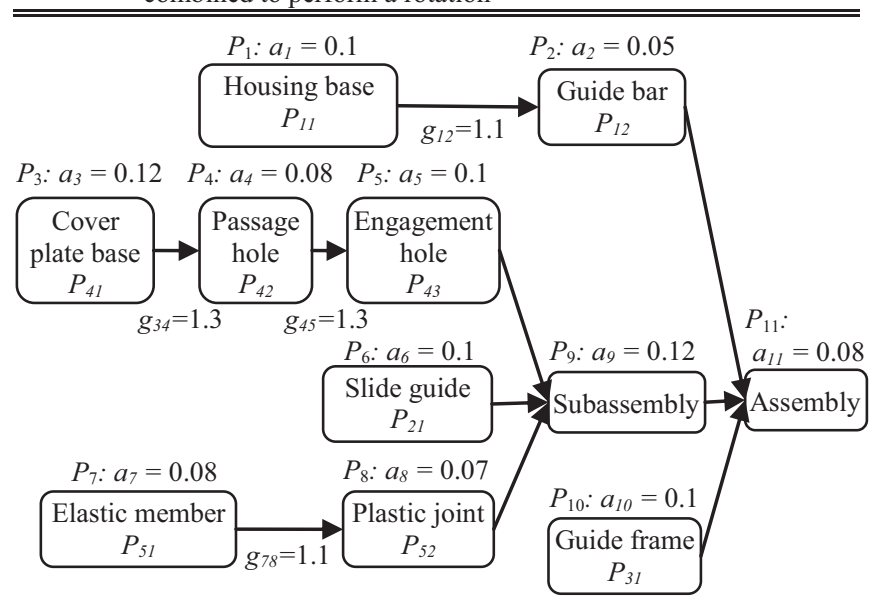

Fig. 5 Manufacturing processes for the slide hinge mechanism

From a managerial standpoint, all the Pareto-optimal scenarios between scenarios $c$ and $e$ may be attractive candidates. While the final executive decision on which scenario to choose remains subject to qualitative perception, plots such as Fig. 6 provide the decision-maker with a visualization of the risk verses cost tradeoff, as well as the outsourcing allocation to achieve it.

TABLE IV

IP LEAKAGE RISKS FOR THE SLIDE Hinge MeChanism

\begin{tabular}{cccccccccccc}
\multicolumn{11}{c}{ claims } & \multicolumn{11}{c}{ Process } \\
& $p_{1}$ & $p_{2}$ & $p_{3}$ & $p_{4}$ & $p_{5}$ & $p_{6}$ & $p_{7}$ & $p_{8}$ & $p_{9}$ & $p_{10}$ & $p_{11}$ \\
\hline$c_{1}$ & 0.5 & 0.5 & 0 & 0 & 0 & 0.5 & 0 & 0 & 0 & 0 & 1.0 \\
$c_{2}$ & 0.5 & 0 & 0.5 & 0 & 0 & 0 & 0 & 0 & 0.8 & 0 & 1.0 \\
$c_{3}$ & 0.5 & 0 & 0 & 0 & 0 & 0.5 & 0 & 0 & 0 & 0 & 1.0 \\
$c_{4}$ & 0 & 0 & 0.5 & 0 & 0 & 0 & 0.5 & 0 & 0.8 & 0.5 & 1.0 \\
$c_{5}$ & 0 & 0 & 0 & 0.5 & 0 & 0 & 0 & 0 & 0.8 & 0 & 1.0 \\
$c_{6}$ & 0 & 0 & 0.5 & 0 & 0.5 & 0 & 0.5 & 0.5 & 0.8 & 0 & 1.0 \\
\hline \hline
\end{tabular}

TABLE V

Claim Weighting Factors For the Slide Hinge Mechanism

\begin{tabular}{ccccccc}
\hline \hline Claim & 1 & 2 & 3 & 4 & 5 & 6 \\
\hline$w_{k}$ & 0.18 & 0.18 & 0.16 & 0.18 & 0.1 & 0.20 \\
\hline \hline
\end{tabular}

TABLE VI

RisK AND DisCount PARAMETERS FOR THE SLIDE HINGE MECHANISM

\begin{tabular}{ccccccccccc}
\hline \hline Supplier & $\alpha^{\min }$ & $\alpha^{\max }$ & $\beta^{\min }$ & $\beta^{\max }$ & $\mu$ & $\sigma$ & $\eta$ & $\rho$ & $d^{\min }$ & $d^{\max }$ \\
\hline In-House & 0.00 & 0.00 & 0.00 & 0.00 & 0.0 & 0.0 & 0.0 & 0.0 & 0.00 & 0.00 \\
1 & 0.30 & 0.45 & 0.15 & 0.20 & 0.5 & 1.0 & 0.5 & 1.0 & 0.20 & 0.24 \\
2 & 0.20 & 0.30 & 0.10 & 0.17 & 0.5 & 1.0 & 0.5 & 1.0 & 0.16 & 0.20 \\
3 & 0.31 & 0.46 & 0.16 & 0.21 & 0.5 & 1.0 & 0.5 & 1.0 & 0.20 & 0.24 \\
\hline \hline
\end{tabular}

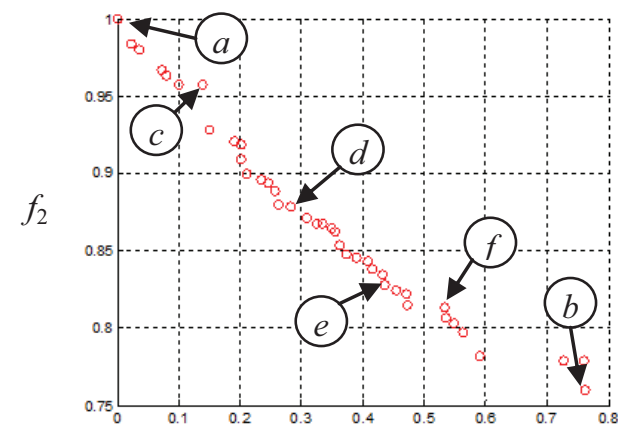

$f_{1}$

Fig. 6 Pareto-optimal outsourcing scenarios for the slide hinge mechanism TABLE VII

SELECTED PARETO-OPTIMAL OUTSOURCING SCENARIOS

\begin{tabular}{ccccccc}
\hline \multirow{2}{*}{ Process } & $a$ & $b$ & $c$ & $d$ & $e$ & $f$ \\
\hline$p_{1}$ & In-House & Sup-1 & In-House & In-House & Sup-2 & Sup-2 \\
$p_{2}$ & In-House & Sup-1 & In-House & In-House & Sup-2 & Sup-2 \\
$p_{3}$ & In-House & Sup-1 & In-House & Sup-2 & Sup-1 & Sup-1 \\
$p_{4}$ & In-House & Sup-1 & In-House & Sup-2 & Sup-1 & Sup-1 \\
$p_{5}$ & In-House & Sup-1 & In-House & Sup-2 & Sup-1 & Sup-1 \\
$p_{6}$ & In-House & Sup-1 & In-House & Sup-2 & Sup-1 & Sup-1 \\
$p_{7}$ & In-House & Sup-1 & Sup-2 & Sup-1 & Sup-3 & Sup-3 \\
$p_{8}$ & In-House & Sup-1 & Sup-1 & Sup-1 & Sup-3 & Sup-3 \\
$p_{9}$ & In-House & Sup-1 & In-House & In-House & In-House & Sup-2 \\
$p_{10}$ & In-House & Sup-1 & Sup-1 & Sup-3 & Sup-1 & Sup-1 \\
$p_{11}$ & In-House & Sup-1 & In-House & In-House & In-House & In-House \\
\hline$f_{1}$ & 0.000 & 0.763 & 0.140 & 0.282 & 0.432 & 0.533 \\
$f_{2}$ & 1.000 & 0.760 & 0.957 & 0.878 & 0.834 & 0.813 \\
\hline \hline
\end{tabular}

\section{V.SUMMARY AND FUTURE WORK}

This paper presented an optimization-based approach to achieve risk-averse outsourcing for intellectual property 
protection. Partial outsourcing was considered as a possible option, where multiple non-IP critical manufacturing processes on the same part can be outsourced to different suppliers while IP-critical processes are kept in-house. The estimated IP-loss was modeled based on the probability of a supplier to compromise manufacturing processes and the probability of such compromises lead to the leakage of IP associated with a claim in the patent document. It was modeled that a supplier can compromise either the processes performed by the supplier or the processes "seen" by the supplier - occurred upstream of the process tree. It was also modeled that a supplier would be more likely to compromise a process if it was performing or seeing multiple processes on the same part. The manufacturing cost was modeled based on the in-house manufacturing cost of each part and the discount rates of each supplier form the in-house costs. As a negative effect of partial outsourcing, it was modeled that outsourcing multiple processes on the same part to different suppliers could increase overall manufacturing cost, by preventing the utilization of net forming processes.

We envision the future work would include:

- Estimation of probability $\omega_{k i}$ based on the natural language processing of patent documents.

- Estimation of $\alpha_{i l}(\boldsymbol{x})$ and $\beta_{i l}(\boldsymbol{x})$ based on the standard best practices for information security such as ISO 27002 [35] and qualitative ranking system such as AHP [11-13].

- Inclusion of process tree as a design variable.

- Inclusion of traditional supplier selection criteria such as part and product quality, lead time, inventory level, volume discount, multiple product types.

- Extension to other types of outsourcing such as software, healthcare, service, and business processes.

\section{REFERENCES}

[1] United States Department of Justice, 2004, Report of the Department of Justice's Task Force on Intellectual Property.

[2] Kumar, S. and Eickhoff, J., 2005/2006, "Outsourcing: when and how should it be done?" Information Knowledge Systems Management vol. 5, pp. 245-259.

[3] Kim, J., Hamza, K., and Saitou, K., 2009, "Optimal outsourcing for intellectual property protection and production cost minimization," Proceedings of the IEEE International Symposium of Assembly and Manufacturing, November 17-20, Suwon, Korea, pp. 124-129.

[4] Coello, C., Lamont, G. and Van Veldhuizen, D., 2007, Evolutionary Algorithms for Solving Multi-Objective Problems, Springer-Verlag,

[5] Dickson, G. W. 1966, "An analysis of vendor selection systems and decisions," Journal of Purchasing, vol. 2, no.1, pp.28-41.

[6] Weber, C., Current, J. R., and Benton, W. C., 1991, "Vendor selection criteria and methods," European Journal of Operational Research, vol. 50 , pp. 2-18.

[7] de Boer, L., Labro, E., and Morlacchi, P., 2001, "A review of methods supporting supplier selection," European Journal of Purchasing \& Supply Management, vol. 7 pp. 75-89.

[8] Weber, C.A., Desai, A., 1996, "Determination of paths to vendor market efficiency using parallel co-ordinates representation: a negotiation tool for buyers," European Journal of Operational Research, vol. 90, pp. 142-155.

[9] Talluri, S. and Narasimhan, R., 2005, "A note on "A methodology for supply base optimization"," IEEE Transactions on Engineering Management, vol.52, no.1, pp. 130-139.

[10] Ng, W. L., 2008, "An efficient and simple model for multiple criteria supplier selection problem," European Journal of Operational Research, vol. 186, no. 3, pp. 1059-1067.
[11] Narasimhan, R., 1983., "An analytic approach to supplier selection," Journal of Purchasing and Supply Management, vol. 1, pp. 27-32.

[12] Bard, J. F. 1992, "A comparison of AHP process with multi-attribute utility theory: a case study," IIE Transaction, vol. 24 no. 5, pp. 111-21.

[13] Ghoudsypour, S.H. and O'Brien, C.O., 1998, "A decision support system for supplier selection using an integrated analytic hierarchy process and linear programming," International Journal of Production Economics, vol. 56/57, No. 1-3, pp. 199-212.

[14] Weber, C. A. and Ellram, L. M., 1992, "Supplier selection using multi-objective programming: a decision support system approach," International Journal of Physical Distribution\& Logistics Management, vol. 23 , no. 2, pp. 3-14

[15] Weber, C. A. and Current, J. R., 1993, "A multiobjective approach to vendor selection," European Journal of Operational Research, vol. 68, pp. 173-184.

[16] Min, H., 1994, "International supplier selection: a multi-attribute utility approach," International Journal of Physical Distribution and Logistics Management, vol. 24, no. 5, pp. 24-33.

[17] Wadhwaa, V. and Ravindra, A. R., 2007, "Vendor selection in outsourcing," Computers \& Operations Research, vol. 34, no. 12, p. 3725-3737

[18] Pirkul, H. and Aras, O. A., 1985, "Capacitated multiple item-ordering problem with quantity discounts." IIE Transactions, vol. 17, no. 3, pp. $206-11$.

[19] Chaudhry, S. S., Forst, F. G., and Zydiak, J. L., 1993, "Vendor selection with price breaks," European Journal of Operational Research, vol. 70, pp. 52-66.

[20] Rosenthal, E. C., Zydiak, J. L., and Chaudhry, S. S., 1995, "Vendor selection with bundling," Decision Sciences, vol. 26, no. 1, pp. 35-48.

[21] Bu!a, F. P. and Jackson, W. M., 1983, “A goal programming model for purchase planning," Journal of Purchasing and Materials Management, vol. 19, no. 3, pp. 27-34.

[22] Degraeve, Z. and Roodhooft, F., 1998, "Determining sourcing strategies: a decision model based on activity and cost driver information," Journal of the Operational Research Society, vol. 49, no. 8, pp. 781-789.

[23] Stamm, C. L. and Golhar, D. Y., 1993, "JIT purchasing: attribute classification and literature review," Production Planning and Control, vol. 4, pp. 273-282

[24] Garg, D., Narahari, Y., and Viswanadham, N., 2004, "Design of six sigma supply chains," IEEE Transactions on Automation Science and Engineering, vol. 1, vo. 1, pp. 38-57.

[25] Birou, L., Stanley, E. F., and Magnan, G. M., 1997, "Integrating product life cycle and purchasing strategies," International Journal of Purchasing and Materials Management, vol. 33, no. 1, pp. 23-32.

[26] Gaonkar, R. S. and Viswanadham, N., 2005, "Strategic sourcing and collaborative planning in Internet-enabled supply chain networks producing multigeneration products," IEEE Transactions on Automation Science and Engineering, vol. 2, no. 1, pp. 54-66.

[27] Narasimhan, R, Talluri, S., and Mahapatra, S. K., 2006, "Multiproduct, multicriteria model for supplier selection with product life-cycle considerations," Decision Sciences, vol. 37, no. 4, pp. 577-603.

[28] Sterlicchi, J., 2008, “Manufacturing IP securely," Information Security, vol. 5, no. 7, pp. 31-33.

[29] Grinols, E. and Lin, H.C., 2006, "Global patent protection: channels of north and south welfare gain," J. Economic Dynamics and Control, vol. 30 , pp. 205-227.

[30] Kennedya, G. and Clarkb, D., 2006, "Outsourcing to China - risks and benefits," Computer Law \& Security Report, vol. 22, pp. 250-253.

[31] Yanlin, S. and Canhua, K., 2008, "Impact of intellectual property protection on foreign direct investment of southern firms," IEEE Symposium on Advanced Management of Information for Globalized Enterprises, pp. 229-233

[32] Sang Ho Lee, "Personal portable device", International Patent, PCT / KR2007 / 001053, 2007.

[33] Sang Ho Lee, Bo Hyung Lee, Seong Ki Seo, "Slide type personal portable device", International Patent, PCT / WO2007 / 117080, 2007.

[34] Deb, K., Agrawal, S., Pratab, A., and Meyarivan, T., 2000, "A fast elitist non-dominated sorting genetic algorithm for multi-objective optimization: NSGA-II," Proceedings of the Conference on Parallel Problem Solving from Nature VI, Paris, France, pp. 849-858.

[35] International Organization for Standardization, 2005, Information technology -- Security techniques -- Information security management systems - Requirements, ISO/IEC 27001:2005. 